\title{
Optimizing Non-invasive Wellness Care for Maximum Impact: Multisensory Meditation Environments Promote Wellbeing
}

\author{
H J Moller ${ }^{1,2,3, *}$, L Saynor ${ }^{2,3}, \mathrm{H} \mathrm{Bal}^{2}, \mathrm{~K} \mathrm{Sudan}^{3}$ \\ ${ }^{1}$ Faculty of Medicine, Knowledge Media Design, Music and Health Research Collaboratory, University of Toronto, Canada \\ ${ }^{2}$ Digital Futures Initiative, OCAD University, Canada \\ ${ }^{3}$ PRAXIS Holistic Health, Canada
}

Copyright (C) 2016 by authors, all rights reserved. Authors agree that this article remains permanently open access under the terms of the Creative Commons Attribution License 4.0 International License

\begin{abstract}
Public health models of wellness care embracing holistic models of mental health are currently needed that are, protocol-driven and have the capacity for standardization without losing a personalized human-centred intention and execution. Increasing evidence is pointing towards the health benefits of leisure: freely chosen, intrinsically motivated and self-directed "flow states", often environment-directed and quite probably with the potential to enact potent changes of consciousness. Our group has been exploring the phenomena of immersive induced "Leisure" and "Wellbeing" in clinical and research endeavours in recent years, allowing for optimized development of both therapeutics and diagnostics to support these efforts. This update offers a review of our optimized wellness care, designed for maximum effectiveness and minimal invasiveness. Optimal leisure experiences are thought to result in enhanced mental wellbeing, positive affect and transformational learning states that carry over into effectively coping with daily routines, stresses and roles. Our group has developed and researched the medically supervised administration of standardized simulated leisure-state meditation experiences in the context of pleasant, hedonic sensory input incorporating multiple sensory channels (visual, auditory, haptic) to promote broad-spectrum wellbeing in mental health care. In this brief report, we report on a novel clinical mental health methodology: TEMM- a technology-enhanced multimodal meditation stress-reduction program with a broad-spectrum mental health benefit, analogous to conventional Mindfulness Based Stress Reduction (MBSR) programs, and a therapeutic risk-benefit margin possibly superior and often preferred by patients to medication therapy attending the PRAXIS holistic health centre. We touch upon seamless diagnostic evaluation and clinical utility of Wellpad, our Electronic Medical Record (EMR) system developed using an iterative Inclusive Design approach. We place our
\end{abstract}

multisensory TEMM meditation therapy within the scope of Virtual Environment Therapy (VET) and suggest the mechanism of action as an induced leisure or flow state to potentiate relaxation, stress-reduction, resilience and personal transformation. The relevance of leisure states to wellbeing and specifically positive experiential learning through inspirational/motivational shifts in consciousness delivered via multimodal immersive environments are described as an important health promotion avenue to pursue and the public mental health research community to consider as new improved, paradigms are developed, aimed at maximizing efficacy and cost-efficiency while minimizing iatrogenic outcomes.

Keywords Meditation, Virtual Reality, Immersive Environment, Leisure, Wellbeing, Stress-reduction, MBSR

\section{Introduction}

It is fair to say that Virtual/Immersive-based therapies have struggled to integrate into mainstream mental healthcare, yet the opportunity in the current era to demonstrate relevance in the healing arts is greater than ever. For some time, there has been promise that media technologies such as VET's could provide effective and standardized health delivery options. [1] Holistic healthcare paradigms incorporating media technology may now play a role in delivering on this promise. In an era where there is a crisis of confidence among the public and academia in scientific reporting of biomedical healthcare studies, [2] informed patients now often seek wellbeing restoration rather than illness treatment as a true healthcare goals [3]; given this trend, the opportunity has arisen for immersive technologies to clinically deliver longstanding health claims 
of representing a pathway towards credible, safe and effective therapeutics. Many patients skeptical of biomedical risk-benefit ratios gravitate to holistic and/or "natural" health and wellness models, such as meditation, yoga, naturopathy, or massage therapy (to name a few). Particularly in mental health care, conventional therapeutic options are often ineffective and/or limited by undesirable iatrogenic side effects, leading to non-compliance. Ideal VET's that seek to improve upon this track record of biomedical therapies need to be salient, aesthetically pleasing and hedonically rewarding, causing patients to seek them out, rather than enduring them through cumbersome tasks or aversive stimuli such as typical VET phobia protocols.

At our health centre, reproducible technology-enhanced multimodal meditation (TEMM) protocols are gradually being developed into therapeutic programs to meet the needs of patients seeking mental health care for safe and effective symptom relief of stress-related symptoms such as anxiety, insomnia and depression. In parallel to this, we are employing inclusively designed diagnostic tools for data capture of clinical outcomes.

As boundaries between real and virtual, technologically mediated and "organic" states of consciousness continue to blur with the march of media technology, the need to address this convergence in a therapeutic paradigm is increasingly relevant and warranted. [4] In parallel to this, the fast pace of technology in work environments, and the impact of this on health is described by Heusser. [5] We have recently advocated for the public health implementation of more accessible leisure opportunities to create a healthier and productive society via personalized immersive and standardized media-based therapeutics. [6] In this sense, while we describe in brief specific clinical therapeutics and diagnostics, it ought to be understood that the public health implications of our report have implications broader than within a clinical context and endeavoring broader public health wellbeing benefits. [6-7]

\section{Immersive Meditation Therapeutics Description}

Our group has developed and researched the medically supervised administration of synthetically "packaged" leisure-state meditation experiences in the context of pleasant, relaxing hedonic sensory input incorporating multiple sensory channels (visual, auditory, haptic). Detailed psychobiological models have been previously reported by the authors. [6-9] As outlined above, the overarching goal of our meditation protocols is to simulate or "recreate" leisure states as per Mihaly Csikszentmihalyi's description of "flow" as an immersive, often hedonic state of absorption and peak performance with positive psychological outcomes. [10] Also described by Revonsuo [11] as "inner presence", this complex consciousness process involves the processing of sensory stimuli and consolidation with previously integrated information, very similar to that described in our neurobiological process of dreaming, [12] and in Csikszentmihalyi's flow model of highly memorable and meaningful peak states, using immersive simulated environments to approximate the best experiences of people's lives.

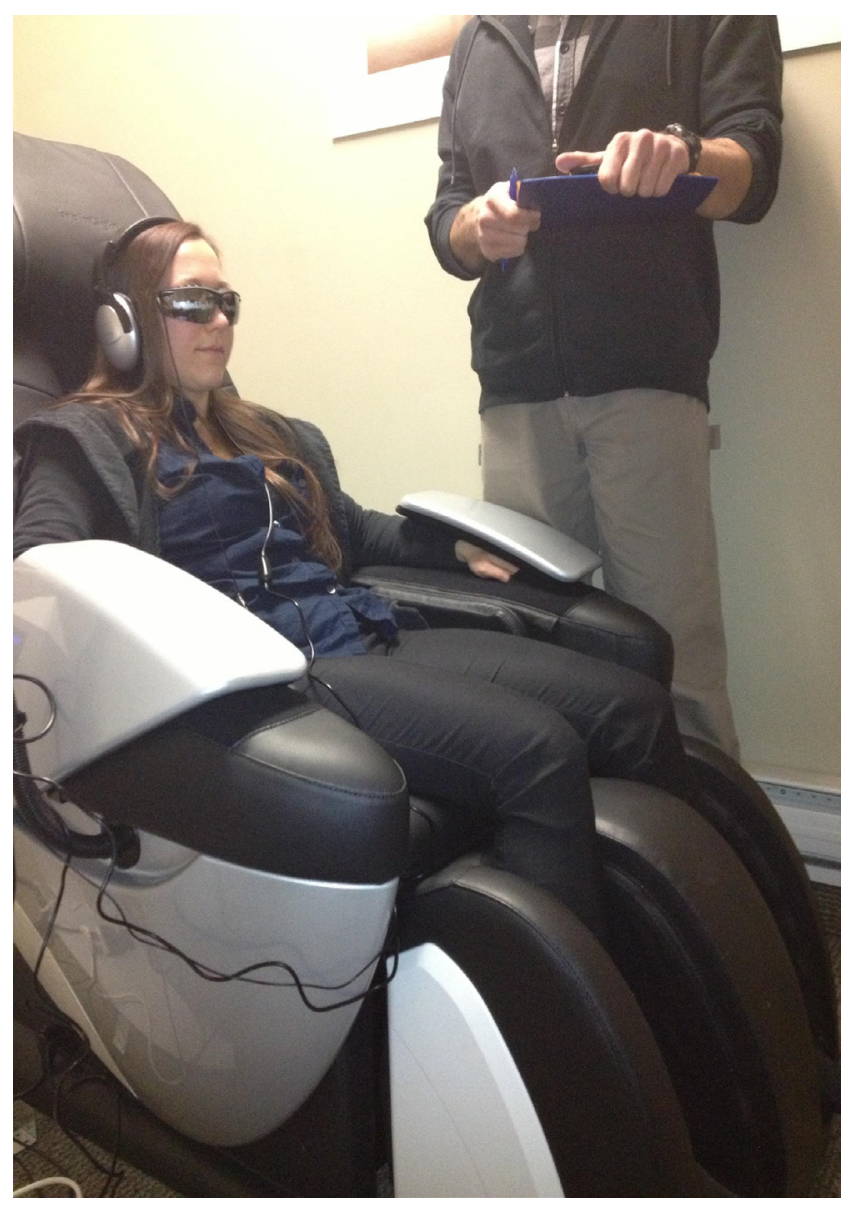

Figure 1. Medically supervised TEMM therapy

We now review briefly a typical clinical protocol for medical patients who have undertaken a standardized course of regularly scheduled (weekly or biweekly) medically supervised 20-40 minute multisensory technology-enhanced multimodal meditation (TEMM) sessions to therapeutically address stress-related symptoms in a psychosupportive paradigm. TEMM's multimodal nature consists of visual, auditory and haptic sensory cues to users. Visual cues in our current TEMM model are recurrent light pulses via specialized glasses using built-in light emitting diodes (LED's), at a frequency between 2 and $12 \mathrm{~Hz}$, corresponding to the electroencephalographic (EEG) rhythm ranging from delta $(1-4 \mathrm{~Hz})$, through theta $(4-8 \mathrm{~Hz})$ and alpha $(8-12 \mathrm{~Hz})$ brain activity, to entrain a calming and relaxed user state, compared to higher frequencies common in chronic high stress-states. The audio component typically involves exposure to a standardized guided meditation invoking a relaxing scenario such as a nature experience (e.g. walking in a meadow or sitting on a beach) accompanied by repetitive positive affirmations and mantras to enhance a participant's 
self-esteem or psychological outlook. Themes addressed within the meditation sessions include "dealing with stress", "relax", "balancing your moods" and "creative problem solving". The intent of the standardized audio content of the immersive TEMM-based meditation scenarios is to mimic, and on a therapeutic level, reprogram autonomous thought processes. Non-invasive haptic sensory stimulation (gentle massage, heat, vibration) occurs through a specialized chair that the patient rests upon during the meditation process. Multimodal visual, auditory and haptic elements are synergistically combined within a therapy session to create an integrative and transformative therapeutic experience (see Figure 1). Pre- and post-treatment core psychosomatic self-states are assessed to track response. [9, 13]

\section{Clinical Program Review}

\subsection{Clinical Study}

In our recently reported clinical study of 20 consecutive fully consenting patients seeking medical meditation in a holistic healthcare centre setting using the above protocol, [9] participants were invited to complete a feedback form in which they were asked to describe the following:

(I) Initial symptoms/ concerns which led to treatment

(II) Overall qualitative impression of the treatment

(III) Overall wellbeing state, using 5-point Likert scales patients were asked to rate

(i) Effectiveness of the TEMM treatment for initial symptoms or concerns

(ii) Adequacy of the duration of sessions

(iii) Adequacy of the number of sessions.

Another set of 5-point Likert scales were also used to allow patients to rate symptom-based self-states before and after the treatment plan, including (i) Tension/Relaxation level (ii) Perceived Stress level (iii) Mood state. Lastly, patients were invited to provide open-ended qualitative feedback regarding additional observations comments.

On average there was a noticeable decline in perceived levels of tension $(p<0.001)$ and perceived stress $(p<0.001)$, before versus after the program, reported by study subjects. Over $50 \%$ of individuals specifically commented on the capacity for TEMM to help them relax and better deal with their stress and anxiety. For changes in mood states of patients there was a similarly positive, though less dramatic shift $(p=0.019)$. The TEMM therapy program was found to be significantly effective in addressing the symptoms and concerns of subjects, with a mean rating of 4.15 points on the 5 -point Likert scale. The layout of the treatment was favorably evaluated, with mean ratings for both individual session and program duration near the "neutral" 3 points on the 5-point Likert scale, i.e. close to the "just right" point. Some individuals also articulated their appreciation of the design of the TEMM program; using different sensory and psychological elements integratively seems to create a complete and powerful wellbeing experience.

As with traditional MBSR meditation therapies, TEMM was consistently reported to have helped initiate an introspective reflective dialogue for a select number of users - these individuals reported more self-awareness of emotions and anxieties and are better able to cope with these emotional states outside of the sessions, implying an experiential shift in consciousness. We have dubbed this transformative clinical outcome as a "vacation effect". Flow-related engagement with the therapy experience across multiple senses was described by many patients as engendering a holistic therapeutic benefit, despite some variation in awareness and recollection of the specifics of the guided meditation they had experienced. This is reminiscent of the residual leisure experience of a vacationer returning from a journey or trip and being able to remember and integrate novel thought patterns and/or behaviours observed and experienced into their daily routine.

\subsection{Ongoing Diagnostics Program}

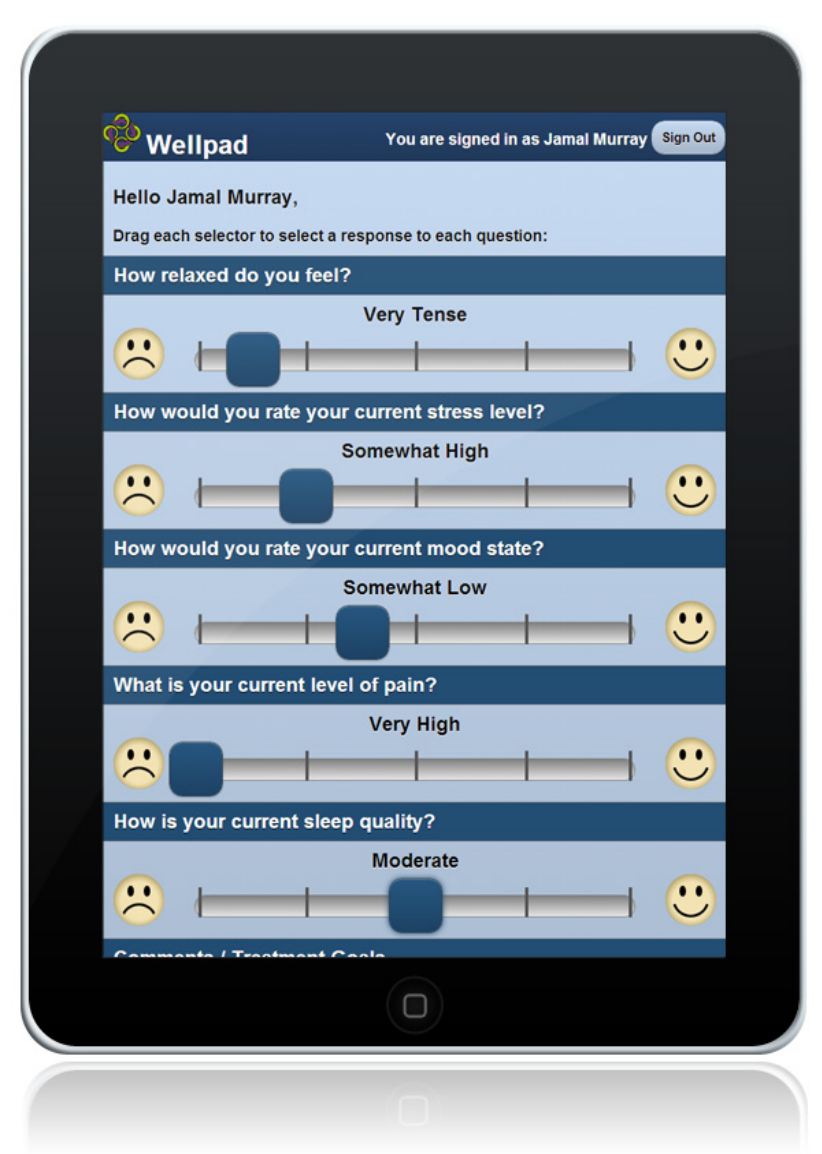

Figure 2. Wellpad EMR Symptom Tracking Tool

A helpful approach to reliably track patient progress is to make this process inclusive, highly usable and even enjoyable for patient and provider. In this sense, to complete a holistic immersive therapeutic experience, the gathering of clinical data should ideally be non-obtrusive and facilitate 
patient-doctor communication across a wide clinical population base. Our tablet-based Wellpad electronic medical record (EMR, see Figure 2) tool represents our team's commitment to Inclusive Design principles described by Nussbaumer [14] and Marti [15] in gathering quantitative and qualitative results by establishing "a universal language that bridges the gap between patient and doctor/circles-of-care, while accommodating the growing reality of diversity in a global community with common health concerns but often differing language and culture." $[13, \mathrm{p} 2]$ Patients of varying cultures, cognitive and physical abilities find self-reporting using Wellpad's novel Happyand Frowny-face sliders to be a significant improvement over complex and confusing paper-based questionnaires, and also report that Wellpad's easy-to-understand data visualization display is helpful to summarize their clinical progress during appointments. [13]

\subsection{Ongoing Therapeutics Development}

A related immersive meditation-based therapy that shows promise for our patient group is vibroacoustic therapy (VAT). Conceptually similar to TEMM, VAT utilizes transducers (loudspeakers), positioned throughout a bed, providing somatic/haptic stimulation with low frequency sound waves, (usually at $40 \mathrm{~Hz}$ or less). While the body is subjected to these low frequency waves, patients are also immersed in binaural auditory music programs delivered via headphones, with pulsating soundscapes comprised of calm and relaxing music. This induces a pleasant wellbeing state for patients, while alleviate pain or reduces nociception. [16] Operationalization and optimization are still core areas of focus for this therapy, but it shows much promise in the further design of multisensory meditative therapies facilitating stress, pain and tension reduction. [8]

\section{Conclusions}

We have described a theoretical framework, case use rationale with early user feedback and public health imperative for standardized therapeutic transformative leisure experiences packaged as immersive media-based meditation programs. We wish to point out the potential societal benefit of public health promotion on a preventative level through TEMM therapy, for example, if more closely linked to workplace health management services. Specifically with difficulty in regularly scheduling predictable vacations or other leisure events, the notion of "bringing leisure into the workplace" may be a promising avenue to pursue for employee wellbeing and productivity. The acute as well as residual effects of such simulated leisure states appears able to approximate real experiences, and perhaps even deliver these more efficiently and predictably. While similar outcomes might ordinarily occur with conventional leisure activities such as play, enjoyment of outdoor nature experiences or cultural activities, it is intriguing to consider that immersive media technologies might be able to operationalize these experiences in a standardized format, allowing essentially a supervised "prescription" of leisure-based multimodal meditation experiences by healthcare professionals trained in this paradigm. This could have significant health policy implications as well as reinventing service delivery in workplace wellbeing initiatives. [17] Clarifying differences between individuals and linking qualitative "lived experience" reports to clinical data would be a welcome next step. The possibilities of personalized experience design of immersive wellbeing environments appear wide open for future work, discovery and implementation.

\section{REFERENCES}

[1] Gregg, L. \& Tarrier, N. (2007), "Virtual reality in mental health: a review of the literature", Social Psychiatry and Psychiatric Epidemiology. 42(5) 343-54.

[2] Pashler, H. \& Wagenmakers, E.J. (2012), "Editors' Introduction to the Special Section on Replicability in Psychological Science: A Crisis of Confidence?" Perspectives on Psychological Science 7: 528-530.

[3] AHHA (2003). Wellness From Within: The First Step, S. Caton (Ed) American Holistic Health Association, Anaheim, CA.

[4] Moller, H.J. (2008), "From Absence to Presence: Blurred consciousness and sleep states", PRESENCE 2008, the $11^{\text {th }}$ Annual International Workshop on Presence, Padova, Italy.

[5] Heusser, M. (2013), "M\&M's, Stress and the Quest for a Sustainable Pace in the Tech Rat Race", Biztech Magazine 8: (Accessible on-line):

http://www.biztechmagazine.com/article/2013/08/mms-stres s-and-quest-sustainable-pace-tech-rat-race

[6] Moller, H.J., H. Bal, K. Sudan, Potwarka, L.R. (2014a), "Recreating Leisure. How Immersive Environments can Promote Wellbeing", In G. Riva, J. Waterworth, D. Murray (Eds) Interacting with Presence: HCI and the sense of presence in computer-mediated environments Degruyter, (Berlin) open access, URL: http://www.presence-research.co $\mathrm{m}$

[7] Moller HJ, Bal, H, Sudan, K, Khan, O. (2014b) Recreating Leisure: How Immersive Therapeutic Environments can Promote Wellbeing. 14th Canadian Congress on Leisure Research. Jerome Singlteon, (ed). Halifax, NS, May 21-24, 2014.

[8] Moller HJ, Bal, H, Sudan, K, Sauer, S. Khan, O. (2014c) Using the Senses to Make Sense. International Association for Music \& Medicine 3rd International Conference, Toronto, Canada June 24-27, 2014

[9] Moller, H.J. Bal, H. (2013), "Technology-enhanced multimodal meditation: Clinical results from an observational case series", Proc 10th International Conference on Virtual Rehabilitation, Philadelphia, PA 26-29 Aug. 2013, pp 1-9. 
[10] Csikszentmihalyi, M. \& Kleiber, D.A. (1991). "Leisure and self-actualization", In B.L. Driver, P.J. Brown, \& G.L. Peterson (Eds.) Benefits of Leisure (pp 91-102). State College, PA: Venture Publishing.

[11] Revonsuo, A. (2006), Inner presence: Consciousness as a biological phenomenon. Cambridge, MA, US: MIT Press.

[12] Moller, H.J. \& Barbera, J. (2006). "Media Presence, Dreaming and Consciousness", In: Riva G, Anguera MT, Wiederhold BK, Mantovani F (Eds.) From Communication to Presence: the integration of cognition, emotions and culture towards the ultimate communicative experience. IOS Press, Amsterdam.

[13] Moller, H.J., Saynor, L. (2014), "Wellpad: An inclusively designed tablet-based digital medical record with optimized efficiency and usability”. MobileHCI 2014, Sept 23-26, 2014, Toronto, Canada.

[14] Nussbaumer, L.L. (2001), Inclusive Design: A Universal Need, Fairchild Books, New York, NY

[15] Marti, P. (2012), Enabling through Design: Explorations of Aesthetic Interaction in Therapy and Care. Technische Universiteit Eindhoven, Eindhoven, Holland.

[16] Wigram, T., Saperston, B. (2013), The Art \& Science of Music Therapy: A Handbook. New York, NY: Routledge.

[17] Peters, T. Ghadiri, A. \& Kohl, M. (2013), "Betriebliches Gesundheitsmanagement: audio-visuelle Entspannung kann Krankenstand senken", CO-MED 19 (4): 40-43. 